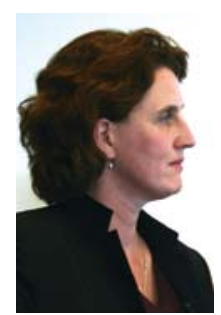

Clara Schlaich
Dr. med. Clara Schlaich, $\mathrm{MPH}$, ist Mitglied des Vorstands der DGMM. Die Leiterin des Hamburg Port Health Center vertritt die Fachgesellschaft im Editorial Board der FTR. Im Jahr 2010 wurde die Geschäftsstelle der DGMM an das Hamburg Port Health Center des Zentralinstituts für Arbeitsmedizin und Maritime Medizin (ZfAM), Direktor: Prof. Dr. Xaver Baur, verlagert. Dr. Schlaich wurde Ende 2006 zur Leiterin Hamburg Port Health Center berufen und ist in dieser Funktion auch Vorsitzende des Arbeitskreises der Küstenländer für Schiffshygiene. Sie ist Fachärztin für Innere Medizin, Infektiologie, Arbeitsmedizin und Suchtmedizinische Grundversorgung. Sie hat an der Johns Hopkins Universität in
Baltimore USA den Master of Public Health erworben. Neben ihren klinischen Weiterbildungsjahren an der Medizinischen Universität zu Lübeck, dem Forschungszentrum Borstel, dem Cook County Hospital in Chicago, Illinois, USA und am ZfAM, war sie als Seuchenreferentin des Landes Hamburg tätig.

Frau Dr. Schlaich ist Mitglied der Deutschen Gesellschaft für Maritime Medizin, der International Maritime Health Association und Vorsitzende der Nationalen Kommission für Infektionsepidemiologie am Robert Koch-Institut. Sie ist regelmäßig als Beraterin der Weltgesundheitsorganisation tätig.

Die besonderen Interessengebiete der Mutter von 4 Kindern sind der internationale Seuchenschutz im Reiseverkehr, an internationalen Grenzübergängen und die gesundheitliche Fürsorge von Seeleuten an Bord und in den Häfen.

\title{
DGMM-Einführungslehrgang „Maritime Notfallmedizin“
}

Am 18. und 19. November 2011 veranstaltete die DGMM erstmals in Eigenregie den Einführungslehrgang „Maritime Notfallmedizin“ am Hamburg Port Health Center des Zentralinstituts für Arbeitsmedizin und Maritime Medizin in Hamburg. Der Lehrgang basiert auf den Empfehlungen eines DGMM-Workshops in Warnemünde im Mai 2006.

Am 8. und 9. März 2007 wurde der Lehrgang erstmalig nach diesen DGMM-Empfehlungen von der Akademie für Rettungsdienst und Gefahrenabwehr der Landesfeuerwehrschule Hamburg mit gutem Erfolg durchgeführt. Hauptzielgruppe waren diejenigen Kollegen, die in Rettungsdiensten mit seewärtigem Auftrag tätig waren.

Die Empfehlungen des Workshops und die Erfahrungen aus dem Pilotlehrgang wurden im folgenden Jahr zu einem Curriculum für ein ,Seminar Einweisung in die Maritime Notfallmedizin' kondensiert (s. DGMM-Homepage, Publikationen/Leitlinien). Dies wurde der Hamburger Landesfeuerwehrschule für deren für März 2009 erneut geplanten Einführungslehrgang zur Verfügung gestellt, der jedoch infolge einer nicht hinreichenden Teilnehmerzahl leider nicht zustande kam.
Die Jahre 2009 und 2010 vergingen mit der leider vergeblichen Suche nach anderen Kooperationspartnern für die Durchführung. So entschied sich die DGMM für die Durchführung in Eigenregie.

Mit der Fachtagung Kreuzfahrtmedizin im Oktober 2010 erschloss sich der DGMM eine neue Klientel, die ein Interesse an einem entsprechenden Fortbildungsangebot bekundete. Aus diesem Bereich stammte dann auch der überwiegende Anteil der Teilnehmer unserer Veranstaltung im November 2011 in Hamburg. Schon kurz nach der Ankündigung zeichnete sich eine Überbuchung ab. Angesichts der Räumlichkeiten und des erwünschten Lernerfolgs war die Teilnehmerzahl auf 25-30 begrenzt. Wir hatten am Ende doppelt so viele Anmeldungen, sodass die DGMM der Wiederholung des Lehrgangs 2012 mit Zuversicht entgegen sieht.

Unter Federführung unseres Vorstandsmitglieds Frau Dr. Clara Schlaich verbuchte das Organisationsteam des HPHC mit Vorbereitung und Durchführung der Veranstaltung einen großen Erfolg, der sich in erfreulich guten Bewertungen der Teilnehmer niederschlug (Gesamtbewertung: $7 \times$ Note $1,10 \times$ Note 2 und $2 \times$ Note 3). Hierfür sei insbesondere Frau Rita

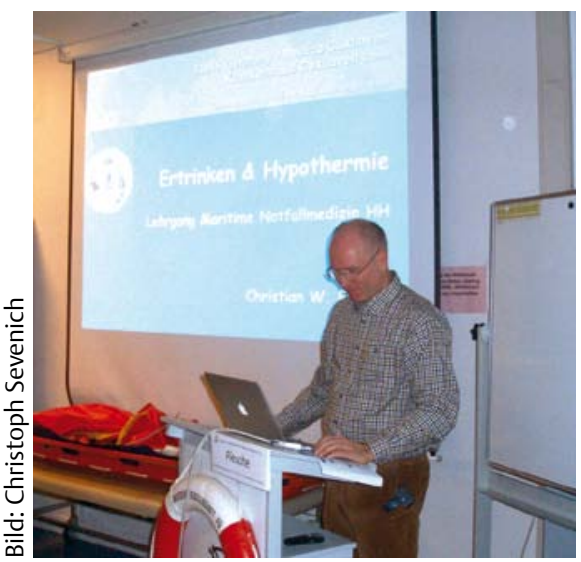

Siebert und Herrn Dipl. Ing. Christoph Sevenich gedankt!

Natürlich gab es auch eine Menge Anregungen für das Curriculum: Neben der Entwicklung eines Kursskripts wird uns vor allem die Frage beschäftigen, ob und wie das Curriculum, welches ursprünglich auf Angehörige von Rettungsdiensten abzielte, auf den Fortbildungsbedarf von Ärzten auf Passagierschiffen ausgerichtet werden kann.

Der Erfolg beruht nicht zuletzt auf engagierten und kompetenten Vortragenden. Auch Ihnen an dieser Stelle nochmals herzlichen Dank!

Dr. Klaus-Herbert Seidenstücker 